\title{
Second-generation $\kappa$-opioid agonist as a peripherally-restricted analgesic
}

Elizabeth H. Schneider ${ }^{1}$; Tyler C. Beck2; Thomas A. Dix ${ }^{2,3}$

1Department of Chemistry - Erskine College; ${ }^{2}$ Department of Drug Discovery and Biomedical Sciences Medical University of South Carolina; ${ }^{3}$ JT Pharmaceuticals, Inc.

Abstract

Today, $\mu$-opioids are prescribed for peripheral pain, but they come with the risk of many toxicities including gastrointestinal issues, sedation, and addiction. The compound of study, referred to as JT09, has shown peripheral pain alleviation with high selectivity of $\mathrm{k}$-opioid receptors compared any effect on the central nervous system as it cannom having blood brain barrier. JT09 has been incorporated into ProNeura rods, subdural implants that continually administers the drug for up to 12 months. This technology may render the need for oral and intravenous administration unnecessary, as well as decrease the number of addictive drugs available to the public. Rats were implanted with the ProNeura rods and blood samples were taken at various time intervals. Results are expected to ProNeura platform and not couse symptoms like in the ProNeura plattorm and not cause symptoms like other
medications that cross the blood-brain barrier. Future directions include pharmacokinetics studies, as well as immunohistochemistry studies to observe the location, action, and relation of $\mathrm{k}$-opioid receptors (KORs) with myocardial infarction (MI) size and its influence on other antigens.

Introduction

The most common reason patients seek medical attention is because of chronic pain ${ }^{1}$, and over-prescription of $\mu$-opioids has led to a major public healh crisis. However, secondgeneration $k$-opioids have been shown to treat chronic and causing the toxicities mediated by opioid receptors of the central nervous system (CNS). ${ }^{2}$ Three receptors mediate opioid analgesic effects: $\delta$, $\mu$, and $\kappa$. $\kappa$-opioid agonists (KOAs), like JT09, have yet to exhibit any of the negative effects of other opioid agonists, and have been shown to be the most efficacious of the opioid receptor agonists in visceral pain models. Studies have supported $J T 09$ as an orally-active peride effects of current opioid andgesics? ProNeura is a technology that has the potential for continuous subcutaneous drug delivery on an outpatient basis or time periods of six to twelve months. JT09 is uniformly distributed throughout the rod, an ethylene vinyl acetate copolymer (EVA) matrix. This allows for controlled release of the
compound as there is no reservoir and no risk of drug dumping. This technology is expected to be a good option for the is repetitive intravenous or oral administration.

Non-addictive orally-active kappa opioid agonists for the treatment of peripheral pain in rats

Therapeutic Potential of Kappa Opioid Agonists

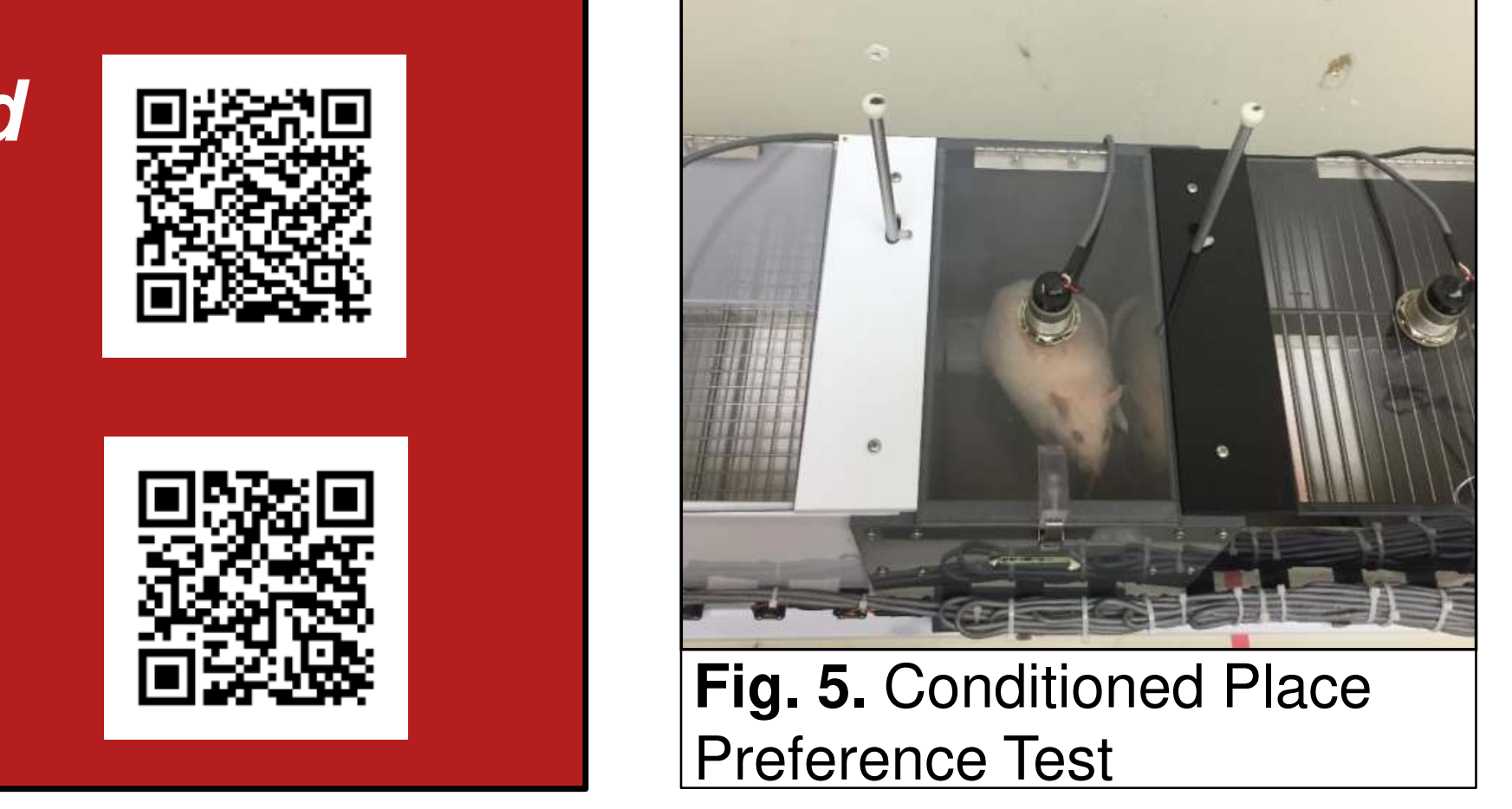
housed in a 12-hour
Methodology

Pain and Behavioral Assessments

Male Sprague Dawley rats (Harlan) were individually

Application of the proprietary JT Pharmaceutical nonnatural amino acid technology to CR665 produced orally active derivatives. The Position 4 D-Arg residue of CR665 was converted to derivatives containing modified D-Arg or D-Lys residues (Figure 1).

Pain assessments included acetic acid-induced rat wehavior preference test, locomotor test, and forced swim assay.

\section{ProNeura Administration}

The ProNeura rods containing JT09, supplied by Titan Pharmaceuticals, were inserted into the dorsal skin in the neck area on three male and three female rats. ${ }^{4}$ Blood samples were taken from the jugular vein cannulation of the rats at various time points, seen in Table

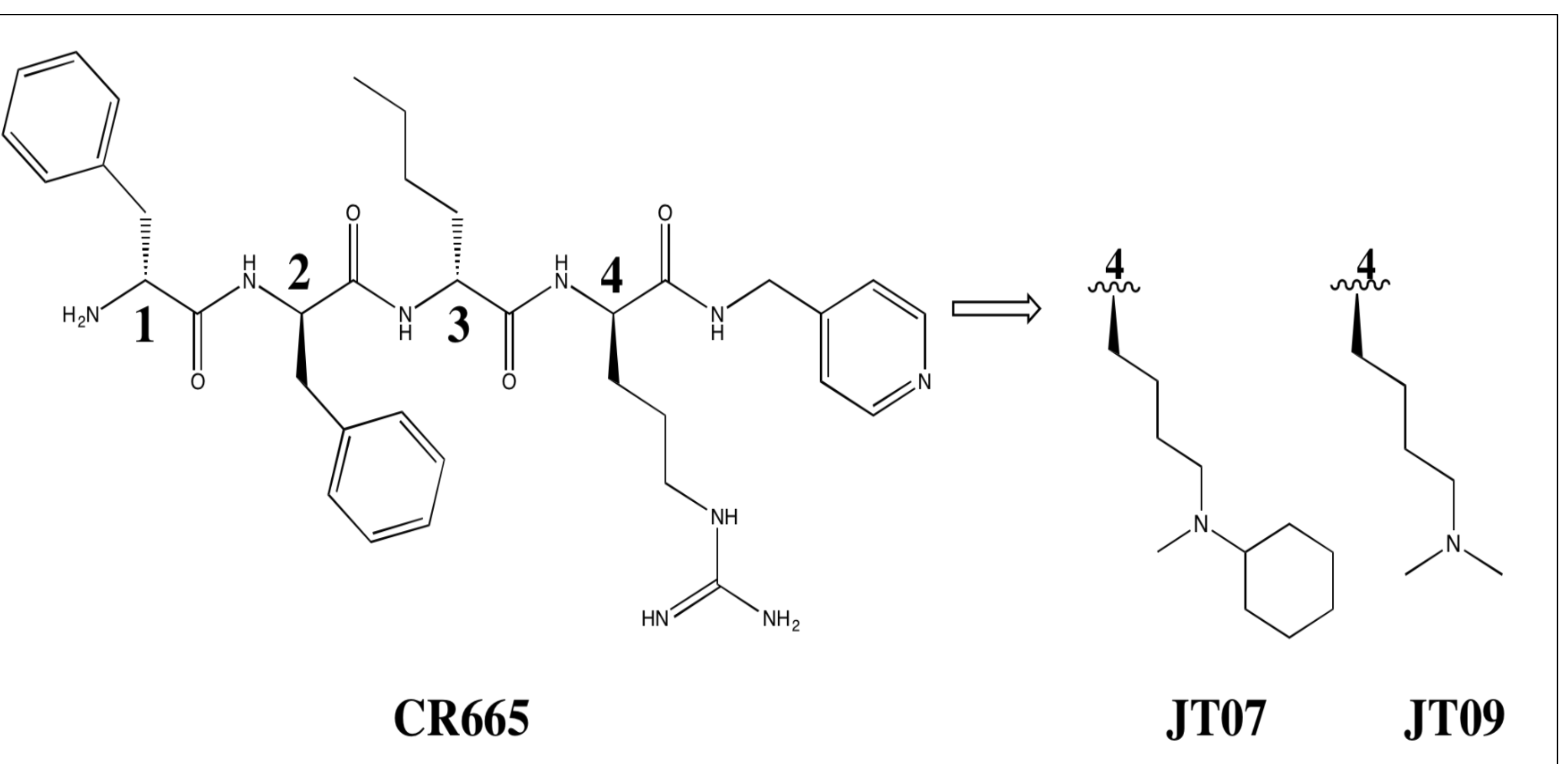

Fig. 1. Structure of CR665 and sidd-chain structures of proprietary CR665 derivatives
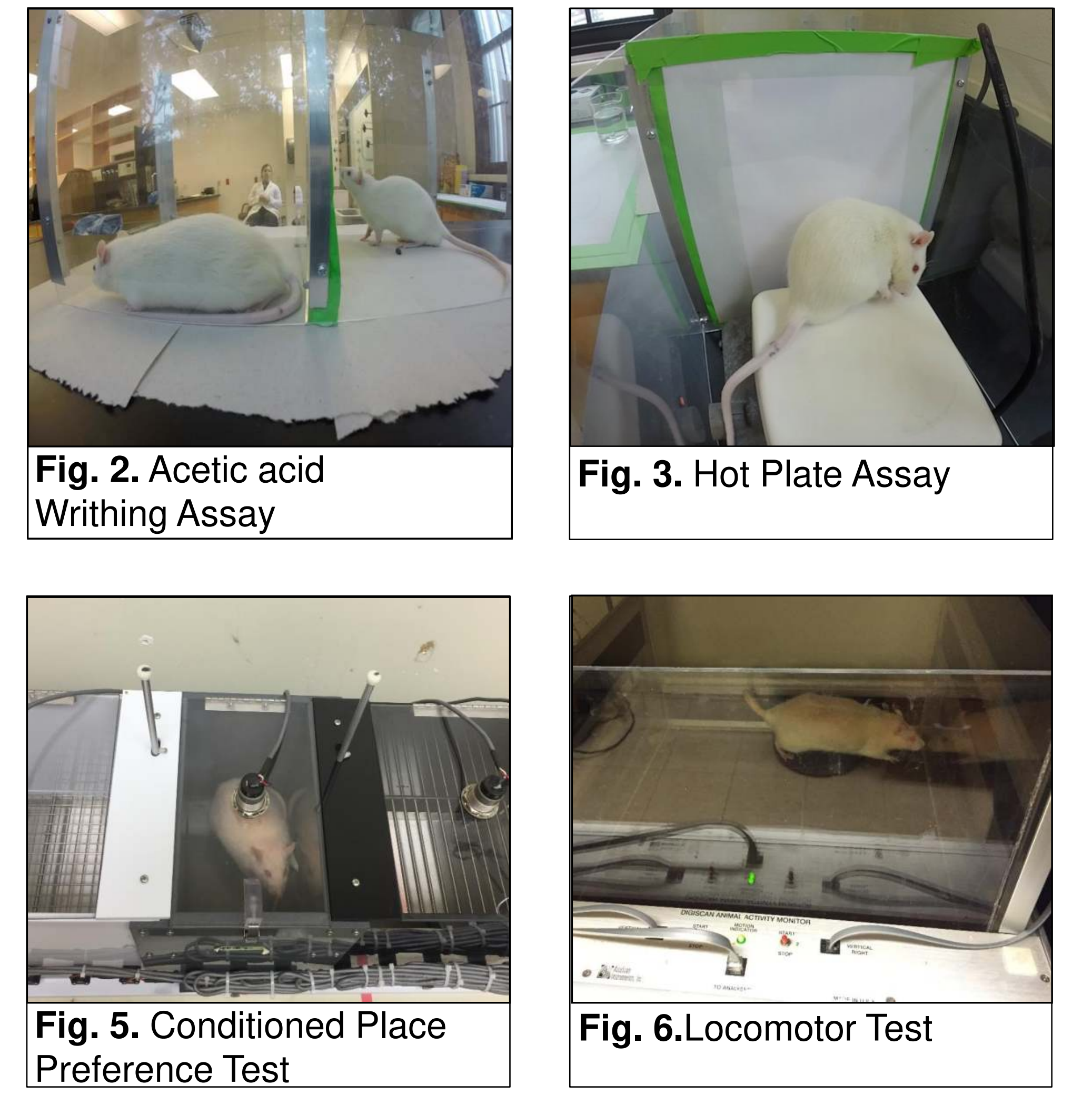

Fig. 4. Self-Administration

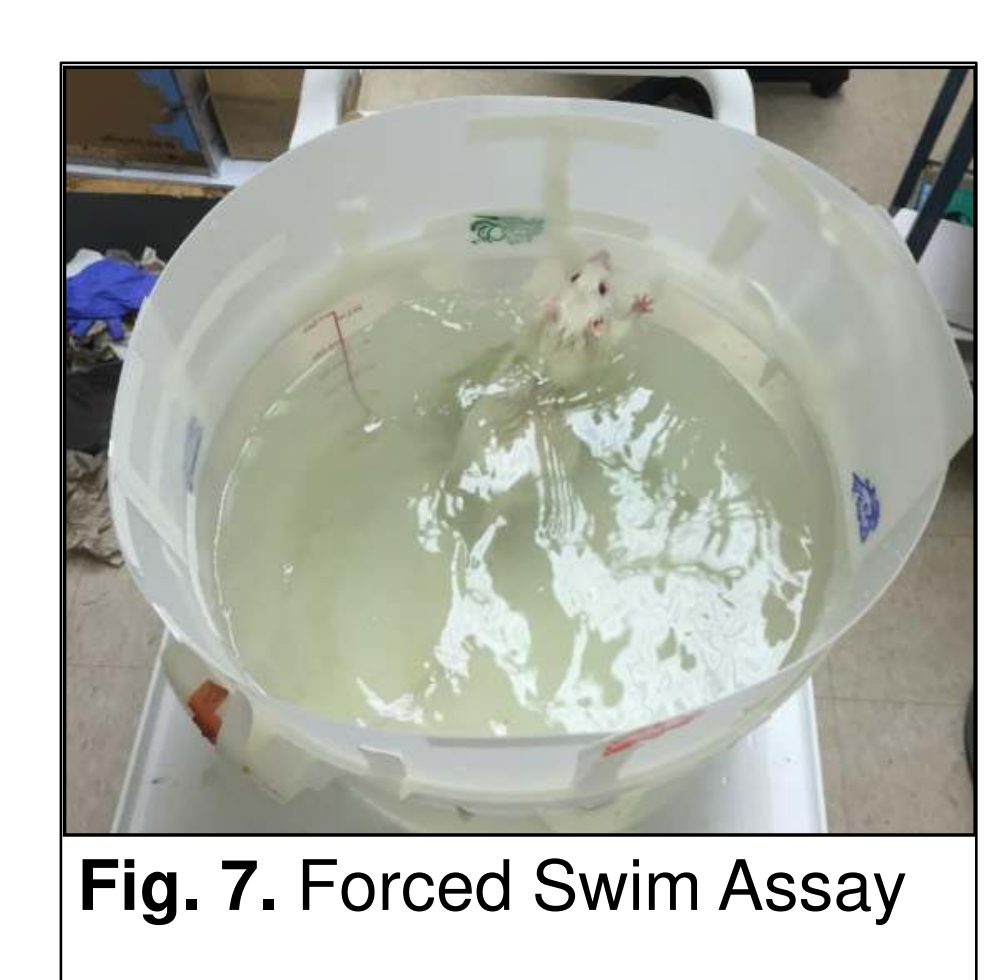

Results

Pain and Behavioral Assessments
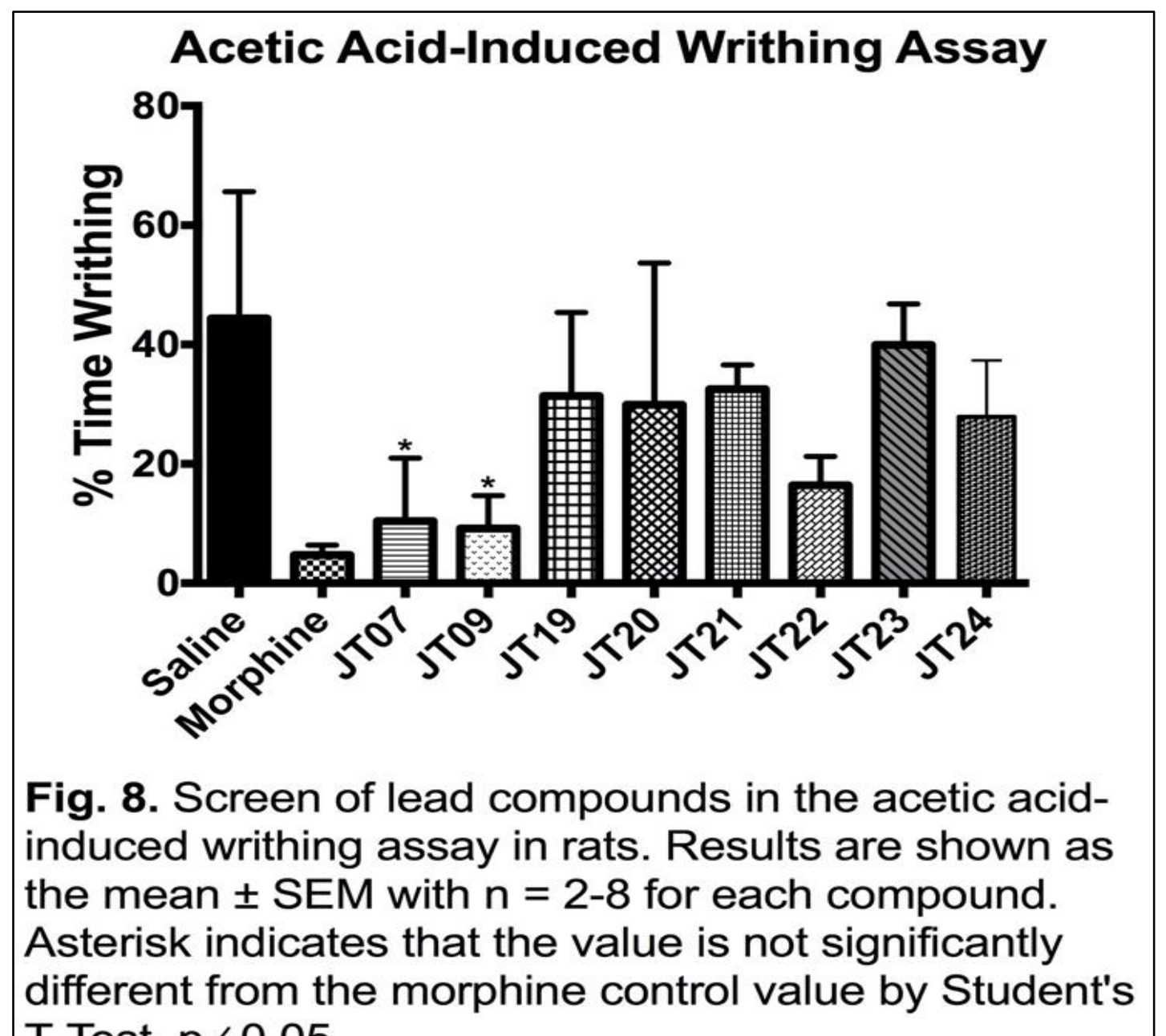

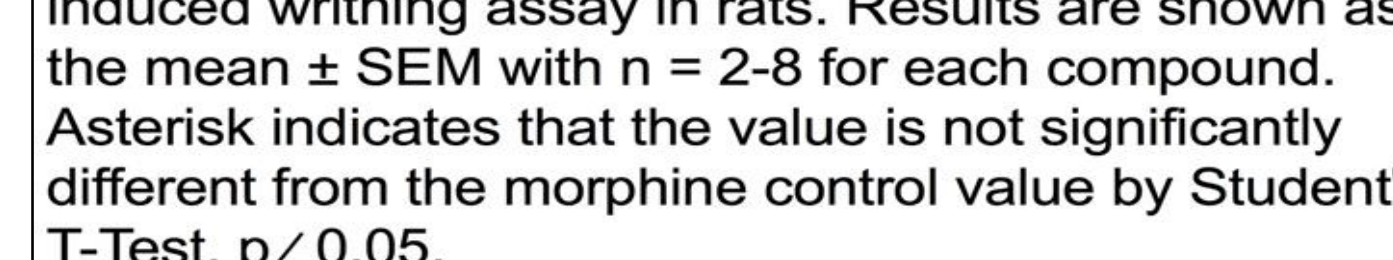

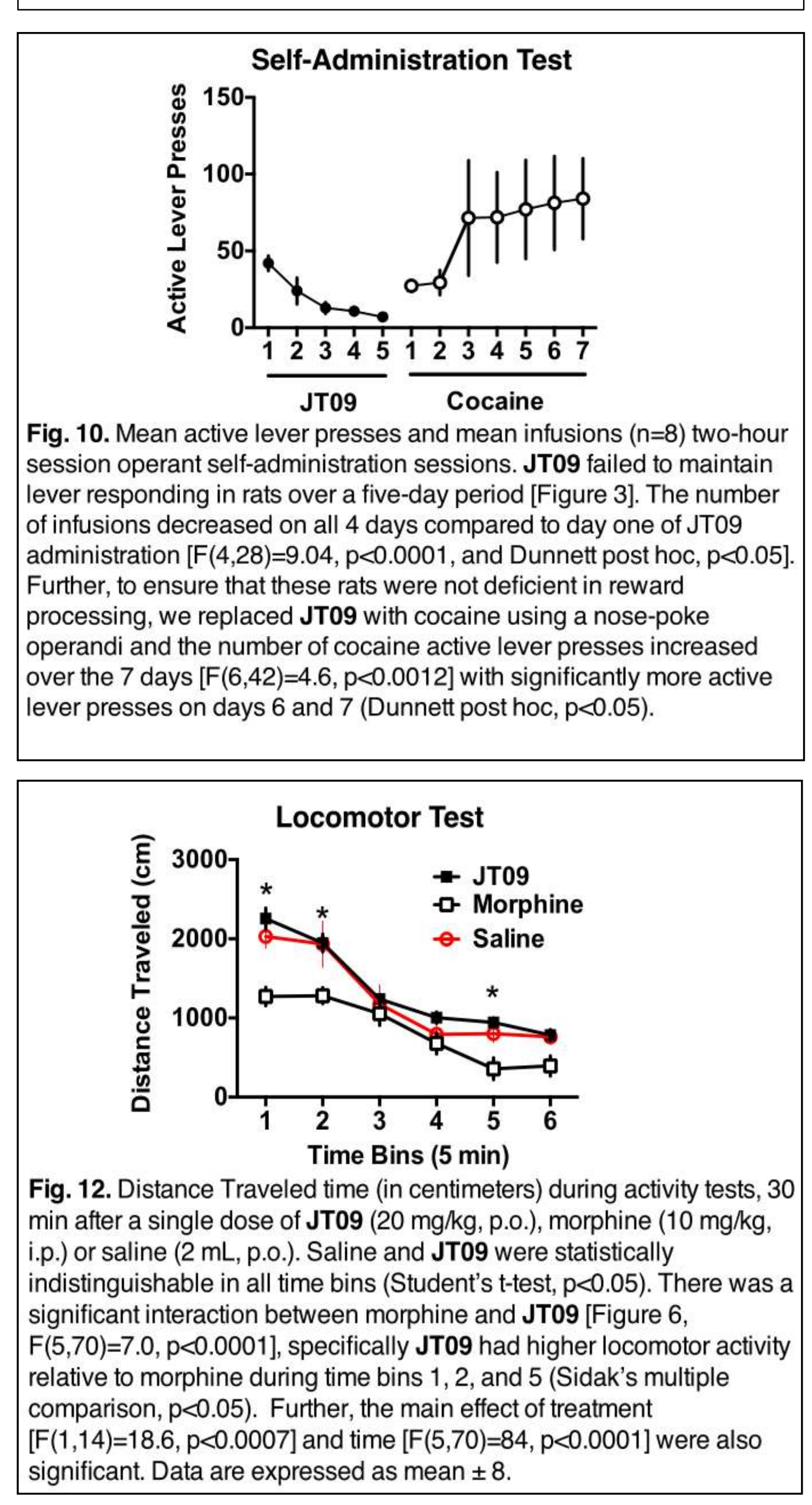

sinnicant Data are xxoessed as mean \pm 8 .

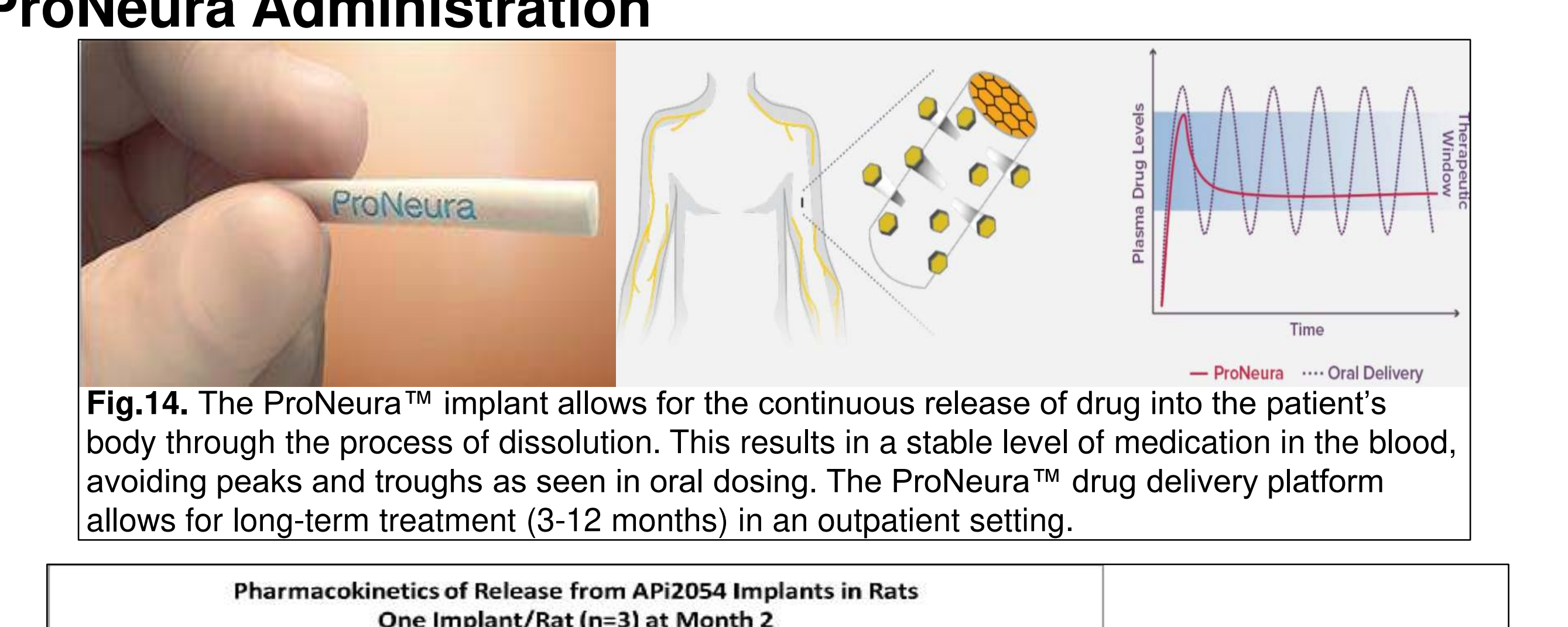

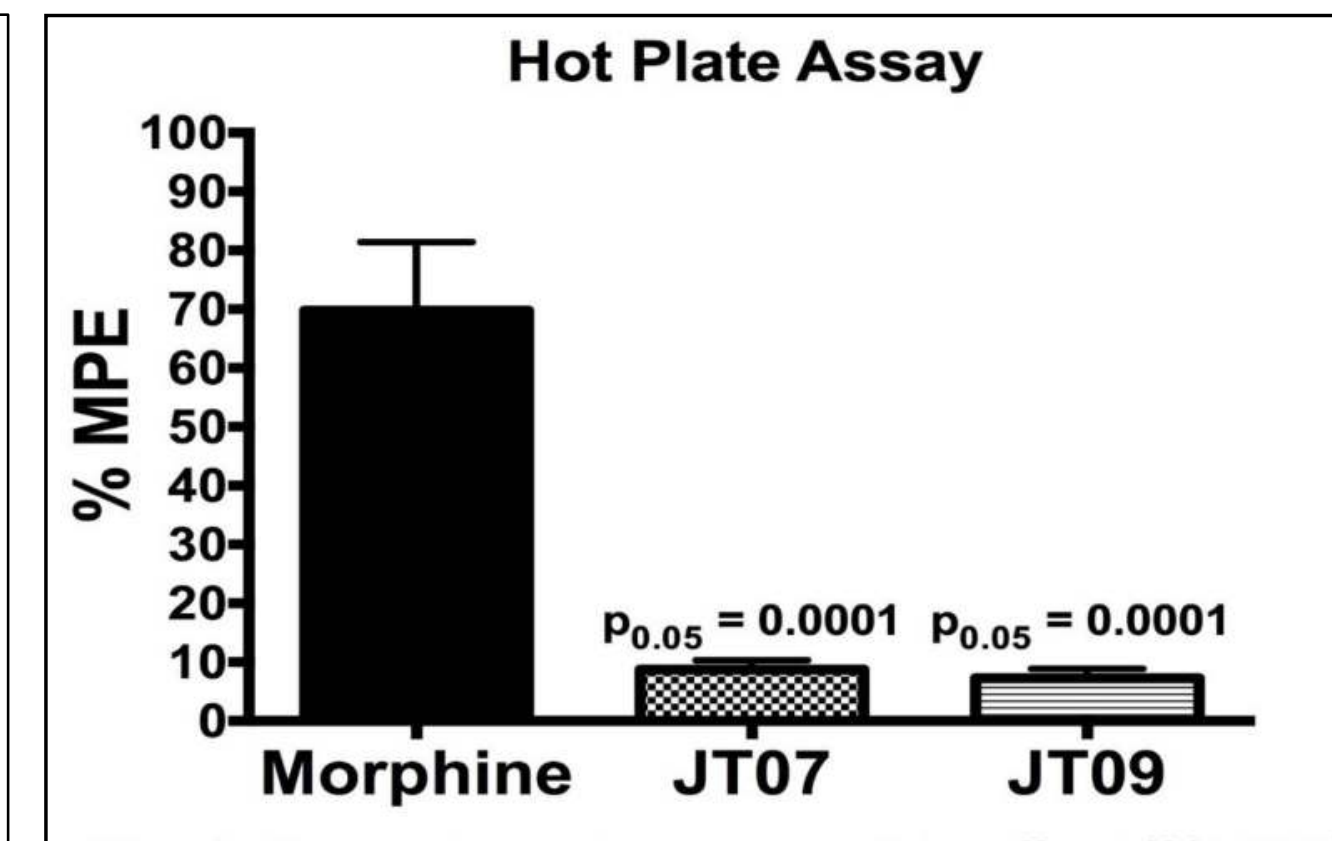
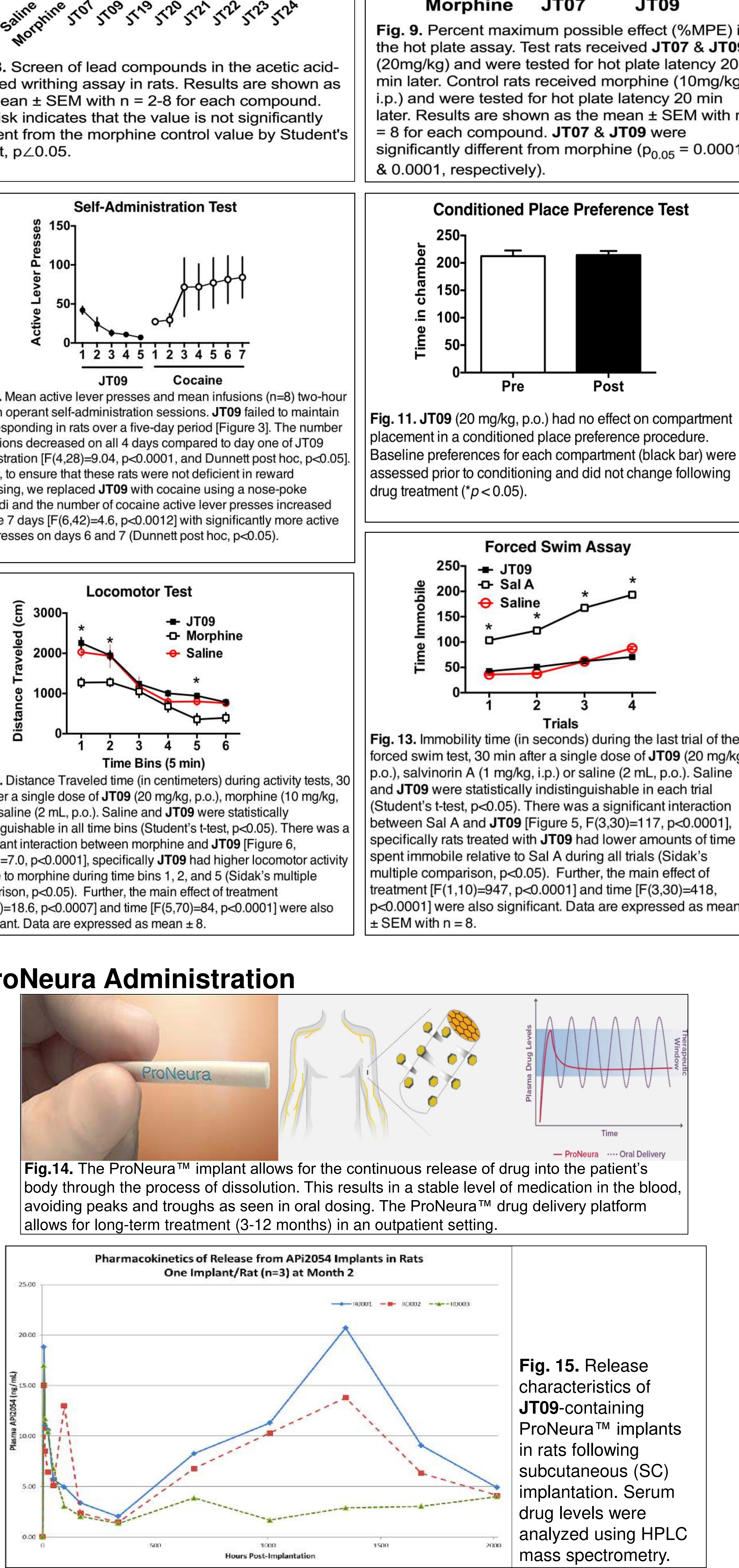

Conclusion

The compound of study, JT09, is as efficacious as morphine in alleviating peripheral pain, while failing to produce undesired CNS-mediated activity such as sedation, dysphoria,
tolerance, and addiction. There were no gross abnormalities in a necropsy after completing a 14-day multiple dose study. ${ }^{1}$ a necropsy after completing a 14-day multiple dose sludy.
JT09 is both orally active and acts as a peripherally restricted $k$-opioid agonist, thus fitting the criteria of a new pain

Efficacy of the compound in the ProNeura platform also allows for a new method of administration for chronic pain JT09 may also be a candidate for prevention and treatment of cardiovascular disease and events.

\section{Future Directions}

Pharmacokinetics studies

JT09 has shown to be orally available in rats; however, the gut environment of humans is much harsher and may determine if JT09 will have the potential of being administered orally in humans and how to optimize delivery. In vivo protocol will be performed to isolate the peptide in serum for HPLC analysis. Previous studies with maximum tolerated dose have given no evidence of toxicity from JT09.

Immunohistochemistry

Immunohistochemistry was used to assess whether k-opioid receptors are co-localized on macrophages, fibroblasts, Immunohistochemistry studies have shown that the kappa opioid receptor is present and active in response to myocardial infarctions. Continued research will be executed to test the application of the compound to treat cardiovascular disease.

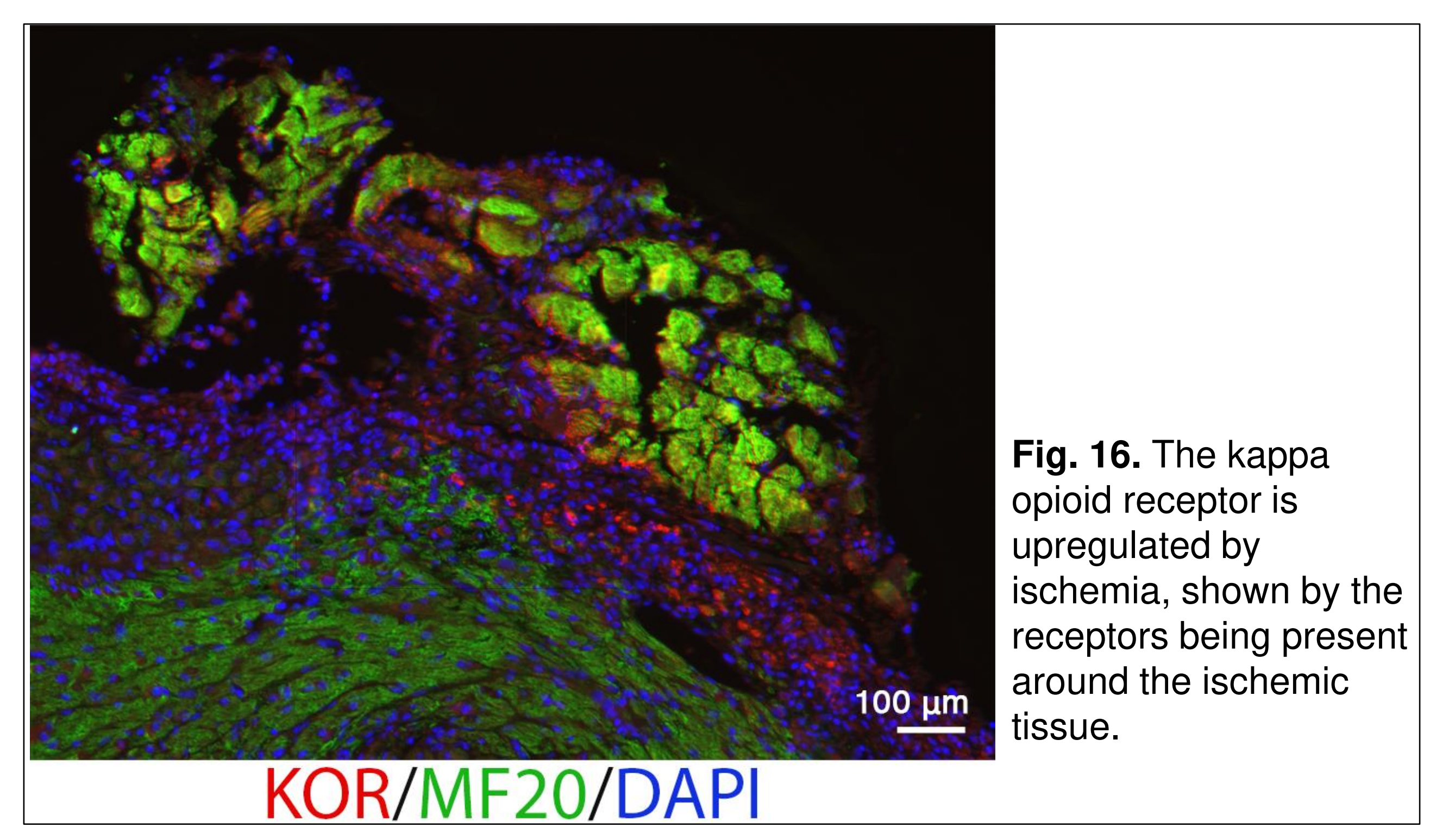

References

Beck, Tyler C.; Hapstack, Matthew A.; Beck, Kyle R.; Dix, Thomas A.
herapeutic Potential of Kappa Opioid Agonists. Pharmaceuticals. 2019. 12. 2Beck, Tyler C.; Reichel, Carmela M.; Helke, Kristi L;; Bhadsaave, Sanat S.; Dix, Thomas A. Non-addictive orally-active kappa opioid ager
peripheral pain in rats. Eur. J. Pharmacol. 2019.8 856 .

${ }^{3}$ Titan Pharmaceut
(accessed 2019). 